\title{
Assessment of the anthocyanidin content of common fruits and development of a test diet rich in a range of anthocyanins
}

\author{
Giulia Corona $^{\mathrm{a}, *}$, Fiona Tang ${ }^{\mathrm{a}}$, David Vauzour ${ }^{\mathrm{a}, \mathrm{b}}$, Ana Rodriguez-Mateos ${ }^{\mathrm{a}}$ and Jeremy P.E. Spencer ${ }^{\mathrm{a}}$ \\ ${ }^{a}$ Molecular Nutrition Group, School of Chemistry, Food and Pharmacy, University of Reading, Reading, UK \\ ${ }^{\mathrm{b}}$ Norwich Medical School, Faculty of Medicine and Health Sciences, University of East Anglia, Norwich, UK
}

Received 15 March 2011; accepted 2 June 2011

\begin{abstract}
There is significant interest in the beneficial health properties of anthocyanins/anthocyanidins. Future investigations into their physiological effects will require human intervention with precise amounts of anthocyanins/anthocyanidins. We have investigated the total anthocyanin/anthocyanidin content of common berry fruits and designed an intervention diet intended to deliver a balanced profile of different anthocyanidins. Using acid hydrolysis and HPLC-diode array analysis we report the individual profile and total anthocyanidin profile/content of blueberry $(148.76 \mathrm{mg} / 100 \mathrm{~g} \mathrm{FW})$, blackberry $(76.78 \mathrm{mg} / 100 \mathrm{~g} \mathrm{FW})$, cranberry $(19.30 \mathrm{mg} / 100 \mathrm{~g} \mathrm{FW})$, black grape $(28.09 \mathrm{mg} / 100 \mathrm{~g} \mathrm{FW})$, raspberry $(104.38 \mathrm{mg} / 100 \mathrm{~g} \mathrm{FW})$ and strawberry $(41.09 \mathrm{mg} / 100 \mathrm{~g} \mathrm{FW})$. The measured anthocyanidin content in the various fruits was used to design an intervention diet ( $15 \mathrm{~g}$ of blueberry, $5 \mathrm{~g}$ of blackberry, $30 \mathrm{~g}$ cranberry, $20 \mathrm{~g}$ of grape, $5 \mathrm{~g}$ of raspberry and $25 \mathrm{~g}$ of strawberry; $100 \mathrm{~g}$ mixed fruit portion) capable of delivering high amounts of the six main anthocyanidins.
\end{abstract}

Keywords: Anthocyanins, berries, HPLC, hydrolysis

\section{Introduction}

Anthocyanins are found abundantly in a variety of berries [1-5], including blackcurrant [6-8] and grape [9, 10] and their regular intake has been linked to a reduction in the incidence of many chronic diseases [11], such as cancer [12, 13], cardiovascular disease [14, 15] and neurodegeneration [16-18]. Furthermore, significant inverse associations between the intake of anthocyanin rich foods and all-cause mortality, death due to coronary heart disease, and death due to cardiovascular disease have been reported [19-22]. There are a wide variety of anthocyanins found in plant foods, all of which have a flavylium (2-phenylbenzopyrylium) cation structure, but which vary in their hydroxyl, methoxyl and glycosidic substitutions [23, 24] (Fig. 1). The anthocyanidins most commonly consumed by humans are pelargonidin, cyanidin, delphinidin, peonidin, petunidin and malvidin (Fig. 1), predominately in their glycosylated forms, in amounts up to $200 \mathrm{mg}$ /day [25]. However, this estimate of intake is compromised due to the huge structural diversity of anthocyanins present in the human diet and problems associated with their characterization and quantification in foods [26]. For example, the total number of glycosylated anthocyanins present in berries is estimated to be around 15 times greater than the number of aglycone forms [2]. As such, a full individual anthocyanin characterization and quantification in foods represents a significant analytical challenge.

${ }^{*}$ Corresponding author: G. Corona, Molecular Nutrition Group, School of Chemistry, Food and Pharmacy, University of Reading, Reading RG6 6AP, UK. Tel.: +44 118378 7713; Fax.: +44 118931 0080; E-mail: g.corona@ reading.ac.uk. 


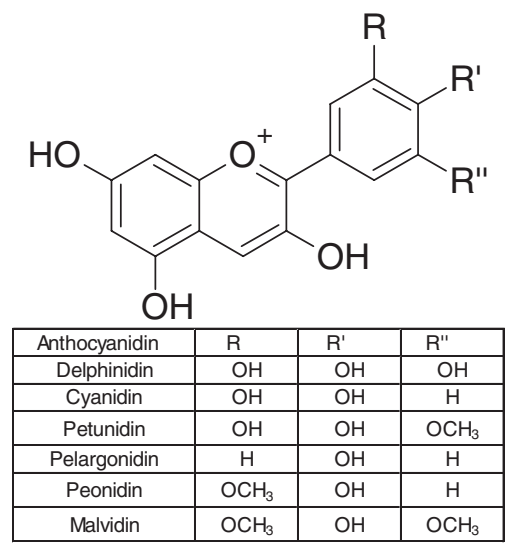

Fig. 1. Chemical structures of major anthocyanidins.

Whilst the focus of many studies has been on the qualitative assessment of the different anthocyanin glycosides present in foods, these studies are not suited to the accurate quantification of their total anthocyanidin content. Such quantitative information is essential for those wishing to conduct well characterized and controlled human intervention studies designed to investigate the impact of anthocyanidin intake on human health. Therefore, we have analyzed a range of berries for their anthocyanidin content using a protocol optimised for the quantification of both anthocyanidins and anthocyanins following their extraction and hydrolysis. We report the anthocyanidin content of the six most commonly consumed berry fruits in the European Union and USA $[1,11]$ and have designed a dietary intervention aimed at delivering balanced quantities of each of the 6 major anthocyanidins. We believe that this diet represents a well-characterized and quantified intervention diet that can be used to fully assess the impact of anthocyanidin intake on human health.

\section{Experimental}

\subsection{Materials}

Commercial UK varieties of blueberry (Vaccinium corymbosum), blackberry (Rubus fruticosus), cranberry (Vaccinium macrocarpum), black seedless grape (Vitis vinifera), raspberry (Rubus idaeus) and strawberry (Fragaria ananassa) were obtained between the periods of July and September. HPLC grade methanol and acetonitrile were purchased from Fisher Scientific UK Limited (Loughborough, UK). Distilled water was obtained from School of Food Biosciences of The University of Reading (Reading, UK). Hydrochloric acid and sodium acetate were purchased from Sigma Chemical Co. (St. Louis, USA). $\beta$-glucosidase from almonds was purchased from ICN Biomedicals Inc., UK. The standards of cyanidin, delphinidin, malvidin, peonidin, petunidin and pelargonidin were obtained from Extrasynthese (Genay, France).

\subsection{Sample preparation}

All fruits were frozen at $-20^{\circ} \mathrm{C}$, freeze-dried for $4-6$ days and ground to a powder on dry ice. Freeze dried powders were then analyzed following the protocol previously described by Nyman et al. [2]. Briefly, samples (10 mg) were extracted and acid hydrolysed using $1 \mathrm{ml}$ of acidified aqueous methanol $(75 \% \mathrm{MeOH} ; 2 \mathrm{M} \mathrm{HCl})$. Extracts were vortexed for $30 \mathrm{sec}$, filtered $(0.45 \mu \mathrm{m})$ and the supernatant retained. After two further extractions of the remaining pellets, samples $(\sim 3 \mathrm{ml})$ were hydrolysed for $50 \mathrm{~min}$ at $90^{\circ} \mathrm{C}$. Following hydrolysis, samples were cooled to room temperature, microfuged at 10,000 $\times \mathrm{g}$ for $3 \mathrm{~min}$ and $50 \mu \mathrm{l}$ was subjected to HPLC analysis. Control experiments were conducted to assess the stability of pure anthocyanidins (cyanidin, delphinidin, malvidin, peonidin, pelargonidin; all $100 \mathrm{mM}$ ) under these processing conditions and indicated that they did not undergo breakdown. 

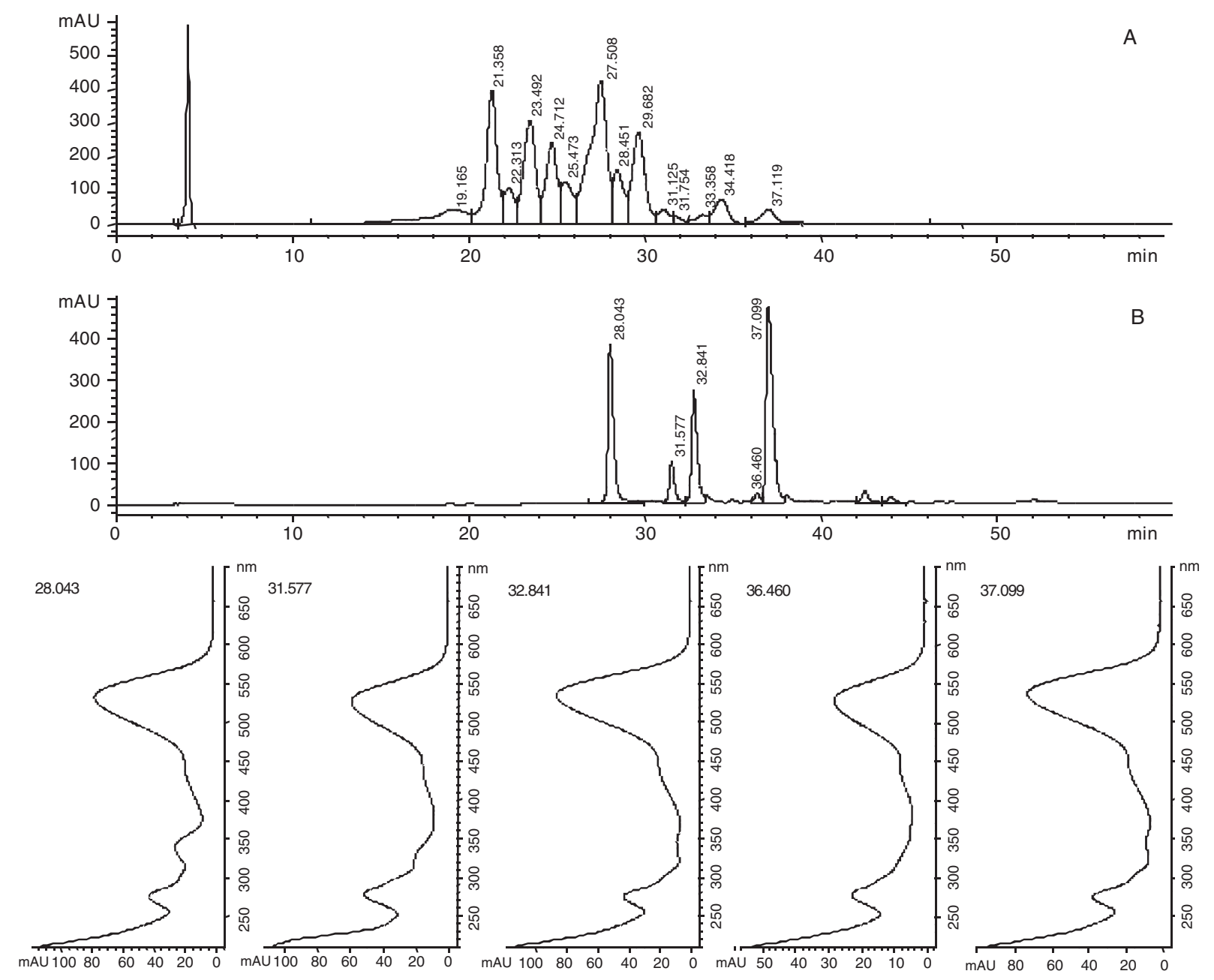

Fig. 2. HPLC chromatograms and spectra of blueberry without hydrolysis (A) and after acidic hydrolysis (B).

Table 1

Parameters of regression equations for calibration curves, limit of detection (LOD) and limit of quantification (LOQ)

\begin{tabular}{lcccccc}
\hline Compound & RT $($ min $)$ & Intercept & Slope & $\mathrm{R}^{2}$ & ${\text { LOD }(\mathrm{uM})^{\mathrm{a}}}$ & ${\text { LOQ }(\mathrm{uM})^{\mathrm{b}}}^{\mathrm{a}}$ \\
\hline Delphinidin & 28.0 & -81.29 & 75.48 & 0.9990 & 0.05 & 0.025 \\
Cyanidin & 31.5 & -85.32 & 114.64 & 0.9994 & 0.025 & 0.05 \\
Petunidin & 32.8 & -86.20 & 93.37 & 0.9992 & 0.05 & 0.025 \\
Pelargonidin & 34.8 & -82.83 & 112.61 & 0.9995 & 0.025 & 0.05 \\
Peonidin & 36.4 & -75.96 & 127.80 & 0.9988 & 0.025 & 0.05 \\
Malvidin & 37.0 & -100.73 & 126.12 & 0.9994 & 0.05 & 0.025 \\
\hline
\end{tabular}

${ }^{\mathrm{a}}$ Concentration yielding a signal-to-noise ratio of $5 ;{ }^{\mathrm{b}}$ Concentration yielding a signal-to-noise ratio of 10 .

\subsection{HPLC analysis}

HPLC analysis utilised a Hewlett-Packard 1100 series liquid chromatograph (Agilent Technologies, Palo Alto, CA) equipped with a diode array detector (HP ChemStation Software system). Separation of samples was achieved 
by RP-HPLC using a Nova-Pak C18 column $(4.6 \mu \mathrm{m} \times 250 \mathrm{~mm} ; 4 \mu \mathrm{m}$ particle size) (Waters) held at a temperature of $30^{\circ} \mathrm{C}$. The mobile phase consisted of a mixture of aqueous methanol $(5 \% \mathrm{MeOH} ; 0.1 \% \mathrm{HCl} 5 \mathrm{M})$ [phase A] and aqueous acetonitrile $(50 \% ; 0.1 \% \mathrm{HCl} 5 \mathrm{M})$ [phase $\mathrm{B}$ ] and was pumped through the column at a flow rate of $0.7 \mathrm{ml} / \mathrm{min}$. The following gradient system was used (min/\% B): 0/5, 5/5, 40/50, 55/100, 59, 9/100, 60/5. The eluent was monitored by photodiode array detection at $520 \mathrm{~nm}$ and spectra of products were obtained over the $200-600 \mathrm{~nm}$ range. Calibration curves of the compounds were constructed using authentic standards $(0.1$ to $100 \mu \mathrm{M})$ and in each case were found to be linear with correlation coefficients of $>0.995$. Before calibration, Linearity and detection and
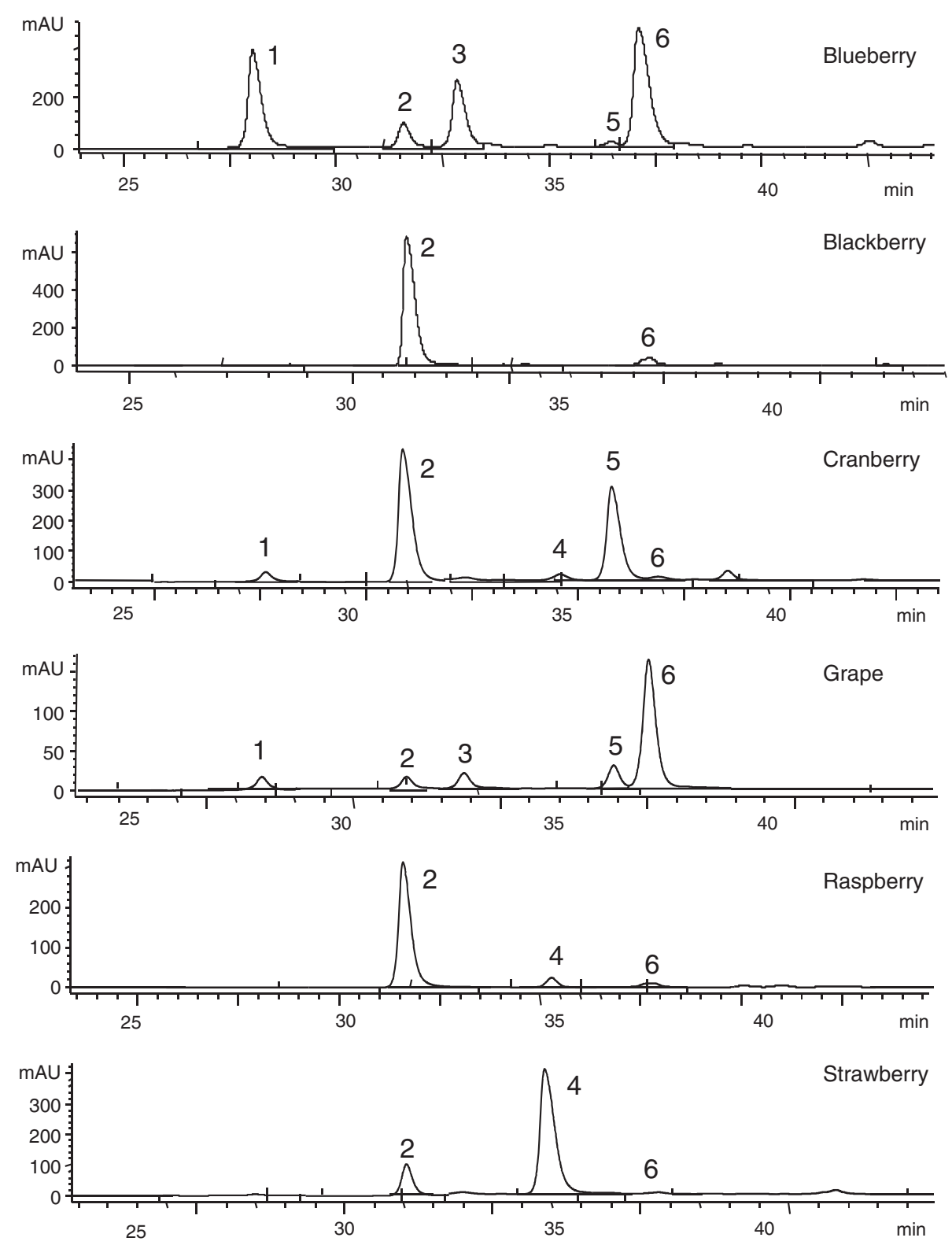

Fig. 3. HPLC chromatograms of blueberry, blackberry, cranberry, grape, raspberry and strawberry extracts after acidic hydrolysis: (1) delphinidin; (2) cyanidin; (3) petunidin; (4) pelargonidin; (5) peonidin; (6) malvidin. 
Table 2

Anthocyanidin contents of different fruits

\begin{tabular}{lcccccrr}
\hline \multicolumn{7}{c}{ Anthocyanidin content $(\mathrm{mg} / 1$} & $00 \mathrm{~g}$ fresh weight) \\
\hline name & delphinidin & cyanidin & petunidin & pelargonidin & peonidin & malvidin & total \\
\hline Blueberry & 56.54 & 8.28 & 29.48 & nd & 2.06 & 52.40 & 148.76 \\
Blackberry & nd & 70.92 & nd & nd & nd & 5.86 & 76.78 \\
Cranberry & 1.08 & 10.29 & nd & 0.56 & 6.92 & 0.45 & 19.30 \\
Grape & 2.91 & 1.57 & 3.35 & nd & 2.86 & 17.40 & 28.09 \\
Raspberry & nd & 91.96 & nd & 7.93 & nd & 4.49 & 104.38 \\
Strawberry & nd & 6.60 & nd & 33.64 & nd & 0.85 & 41.09 \\
\hline
\end{tabular}

nd $=$ non detected.

quantification limits were tested. Limit of detection (LOD) and limit of quantification (LOQ) were defined as the concentration yielding a signal-to-noise ratio of 5 and 10 respectively.

\section{Results and discussion}

In plant foods, anthocyanidins are present as glycosidic forms (anthocyanins) bound to glucose, galactose, arabinose, rutinose, sambubiose, sophorose, sometimes with the addition of an acetyl, caffeoyl, dioxalyl, malonyl, coumaroyl, or succinyl moiety $[2,7,27]$. Direct extraction and analysis of foods for their anthocyanin content often yields complex chromatograms with many unresolved peaks, thereby accurate quantification is difficult. In our study, HPLC analysis of un-hydrolysed fruit samples resulted in chromatograms in which it was difficult to resolve individual anthocyanins and anthocyanidins (Fig. 2A; blueberry as example). In addition, as anthocyanins are relatively unstable, the availability of authentic anthocyanin standards is limited and thus it is virtually impossible to achieve a complete and accurate quantification of the total anthocyanin content via the assessment of anthocyanins alone. In this study we achieved an accurate quantification of the total anthocyanidin content of each fruit via quantification of their major anthocyanidin content post hydrolysis of the extracted fruit. Parameters of calibration curves are reported in Table 1. Acid hydrolysis of the extracted fruits, allowed resolution of individual anthocyanidins (Fig. 2B, blueberry as example), indicating that all the glycosidic forms present in the fruit has been effectively cleaved to yield their respective anthocyanidins. This technique allowed the characterisation of the major anthocyanidins in a number of other anthocyanin-rich fruits (Fig. 3) and allowed quantification of their total anthocyanidin content (Table 2). Cyanidin and malvidin were the most common anthocyanidins as they were present in all fruits tested, whilst petunidin was the least common, present only in blueberry and grape (Table 2). Blueberry, cranberry and grape contained the most diverse array of anthocyanidins with five present in total, whilst blackberry contained the least with only cyanidin and malvidin (Fig. 3; Table 2).

The results obtained in this study were compared with the data available on the USDA Database for the Flavonoid Content of foods (release 2.1, 2007) and the phenol explorer database (Table 3). Generally, our data for each fruit tested were in agreement with the anthocyanidin data reported in both of these databases. For example, our data for blueberry are in agreement with the reported anthocyanidin content for blueberry in the USDA database and slightly higher than the max anthocyanidin value reported in the Phenol Explorer database. The slight differences in the amounts measured in our study and the two databases may reflect differences in the extraction and analysis of fruits [24-27], although differences are also likely to occur due to varietal differences in the fruits analysed, differences in the growing environment, fruit ripeness and storage time [7, 26, 28]. In addition to those anthocyanidin data reported in the two databases, we also observe the presence of malvidin in blackberry and strawberry and pelargonidin in cranberry. Compared to the USDA database, we found higher amounts of cyanidin in raspberry and strawberry and higher amounts of malvidin in raspberry.

The quantification of the total anthocyanidin content in fruit is of particular importance when designing intervention diets for human clinical studies. Indeed, only the use of foods or test materials that have been analytically well characterized and standardized with regard to their flavonoid content and profile will enable significant advancements to be 
Table 3

Anthocyanidin content of tested samples and results from database

\begin{tabular}{|c|c|c|c|c|c|c|c|c|}
\hline \multicolumn{9}{|c|}{ Anthocyanidin content $\mathrm{mg} / 100 \mathrm{~g}$ of fresh weight } \\
\hline \multirow[t]{2}{*}{ Sample } & \multirow[t]{2}{*}{ Anthocyanidin } & \multirow[t]{2}{*}{ Present study } & \multicolumn{3}{|c|}{ USDA flavonoids database $^{\mathrm{a}}$} & \multicolumn{3}{|c|}{ Phenol explorer database ${ }^{b}$} \\
\hline & & & mean & $\min$ & $\max$ & mean & $\min$ & $\max$ \\
\hline \multirow[t]{6}{*}{ Blueberry } & Cyanidin & 8.28 & 16.97 & 4.79 & 48.06 & 6.43 & 1.56 & 13.54 \\
\hline & Delphinidin & 56.54 & 47.4 & 20.82 & 124.16 & 30.22 & 14.52 & 49.28 \\
\hline & Malvidin & 52.4 & 61.35 & 32.95 & 126.44 & 32.34 & 20.40 & 40.86 \\
\hline & Peonidin & 2.06 & 11.38 & 1.01 & 38.99 & 1.17 & 0.25 & 2.23 \\
\hline & Petunidin & 29.48 & 26.42 & 7.19 & 74.89 & 19.34 & 7.94 & 33.01 \\
\hline & Total & 148.76 & 163.52 & 66.76 & 412.54 & 89.49 & 44.67 & 138.91 \\
\hline \multirow[t]{4}{*}{ Blackberry } & Cyanidin & 70.92 & 90.31 & 44.17 & 199.41 & 107.23 & 61.27 & 153.02 \\
\hline & Malvidin & 5.86 & na & na & na & na & na & na \\
\hline & Pelargonidin & nd & 0.15 & nd & 0.51 & na & na & na \\
\hline & Total & 76.78 & 90.46 & 44.17 & 199.92 & 107.23 & 61.27 & 153.02 \\
\hline \multirow[t]{6}{*}{ Cranberry } & Cyanidin & 10.29 & 41.81 & 32.16 & 53.35 & 9.22 & 9.22 & 9.22 \\
\hline & Delphinidin & 1.08 & 7.66 & 0.12 & 10.66 & na & na & na \\
\hline & Malvidin & 0.45 & 0.31 & nd & 1.34 & na & na & na \\
\hline & Peonidin & 6.92 & 42.1 & 32.56 & 58.18 & 23.70 & 23.70 & 23.70 \\
\hline & Pelargonidin & 0.56 & na & na & na & na & na & na \\
\hline & Total & 19.3 & 91.88 & 64.84 & 123.53 & 32.92 & 32.92 & 32.92 \\
\hline \multirow[t]{6}{*}{ Black Grape } & Cyanidin & 1.57 & na & na & na & 0.74 & nd & 4.36 \\
\hline & Delphinidin & 2.91 & na & na & na & 2.04 & 0.32 & 7.44 \\
\hline & Malvidin & 17.4 & na & na & na & 37.45 & 6.57 & 106.92 \\
\hline & Petunidin & 3.35 & na & na & na & 1.85 & 0.03 & 6.31 \\
\hline & Peonidin & 2.86 & na & na & na & 3.94 & 1.38 & 12.82 \\
\hline & total & 28.09 & na & na & na & 46.01 & 8.30 & 137.86 \\
\hline \multirow[t]{5}{*}{ Raspberry } & Cyanidin & 91.96 & 35.84 & 20.73 & 71.11 & 32.23 & nd & 96.09 \\
\hline & Delphinidin & nd & 0.29 & nd & 2.01 & 0.14 & nd & 1.94 \\
\hline & Malvidin & 4.49 & 0.7 & nd & 2.75 & 0.42 & nd & 2.68 \\
\hline & Pelargonidin & 7.93 & 1.85 & nd & 8.23 & 2.90 & nd & 10.28 \\
\hline & total & 104.38 & 38.68 & 20.73 & 84.1 & 35.68 & nd & 110.99 \\
\hline \multirow[t]{5}{*}{ Strawberry } & Cyanidin & 6.6 & 1.96 & nd & 5.42 & 2.98 & 0.93 & 6.02 \\
\hline & Delphinidin & nd & 0.32 & nd & 2.6 & na & na & na \\
\hline & Malvidin & 0.85 & na & na & na & na & na & na \\
\hline & Pelargonidin & 33.64 & 31.27 & nd & 57.49 & 42.52 & 15.34 & 70.45 \\
\hline & total & 41.09 & 33.55 & nd & 65.51 & 45.50 & 16.28 & 76.47 \\
\hline
\end{tabular}

${ }^{a}$ Where the values for individual glycosides were determined, USDA scientists converted the glycoside values into aglycone conversion factors based on molecular weight to make data consistent across the database. ${ }^{b}$ Data from the database have been reported in the table after conversion of the glycoside values into aglycone forms using conversion factors based on molecularweight. nd= non detected; na = non available.

made with regards to the physiological functions of these diets [29]. Methodological differences in sample collection, preparation, chromatographic separation, detection, purity of reference standards, methods of data evaluation, and others result in significant inter-laboratory variations, and represent a limitation with regard to study comparisons and intervention diet design [29]. When added to varietal, agricultural and ripeness differences, we suggest that it is essential that all intervention diets should characterised and quantified prior to their use in human clinical studies in order to facilitate the accurate delivery of precise anthocyanin doses to individuals. We believe that the method reported here represents a rapid and accurate way of achieving this. 




\begin{tabular}{|c|c|c|c|c|c|c|c|c|}
\hline \multicolumn{7}{|c|}{ Anthocyanidin content (mg) } & \\
\hline name & g fresh fruit & delphinidin & cyanidin & petunidin & pelargonidin & peonidin & malvidin & total \\
\hline Blueberry & 15 & 8.48 & 1.24 & 4.42 & 0.00 & 0.31 & 7.86 & 22.31 \\
\hline Blackberry & 5 & 0.00 & 3.55 & 0.00 & 0.00 & 0.00 & 0.29 & 3.84 \\
\hline Cranberry & 30 & 0.32 & 3.09 & 0.00 & 0.17 & 2.08 & 0.14 & 5.79 \\
\hline Grape & 20 & 0.58 & 0.31 & 0.67 & 0.00 & 0.57 & 3.48 & 5.62 \\
\hline Raspberry & 5 & 0.00 & 4.60 & 0.00 & 0.40 & 0.00 & 0.22 & 5.22 \\
\hline Strawberry & 25 & 0.00 & 1.65 & 0.00 & 8.41 & 0.00 & 0.21 & 10.27 \\
\hline B & total (mg) & $\mathbf{9 . 3 9}$ & $\mathbf{1 4 . 4 4}$ & $\mathbf{5 . 0 9}$ & $\mathbf{8 . 9 7}$ & $\mathbf{2 . 9 6}$ & $\mathbf{1 2 . 2 1}$ & $\mathbf{5 3 . 0 5}$ \\
\hline
\end{tabular}

Fig. 4. Design of a fruit mixture containing a balanced amount of all anthocyanidins: (A) HPLC chromatogram of the fruit mixture after acid hydrolysis: (1) delphinidin; (2) cyanidin; (3) petunidin; (4) pelargonidin; (5) peonidin; (6) malvidin. (B) Anthocyanidin content for the fruit mixture containing $15 \mathrm{~g}$ of blueberry, $5 \mathrm{~g}$ of blackberry, $30 \mathrm{~g}$ of cranberry, $20 \mathrm{~g}$ of grape, $5 \mathrm{~g}$ of raspberry and $25 \mathrm{~g}$ of strawberry.

We also used our fruit anthocyanidin quantification data to create an intervention diet that best delivers a balanced profile of the six main anthocyanidins (Fig. 4). The six common anthocyanidins are powerful antioxidants in vitro, and different glycosylation patterns, hydroxyl and methyl groups presence and position, either enhance or diminish the antioxidant power $[23,30]$. In addition, absorption and metabolism in vivo will affect their antioxidant potential [27, $31]$, but health benefits are still reported [27, 31, 32]. To date, most human intervention studies have been conducted with single anthocyanin-rich fruits $[14,15]$, meaning that the biological effects of select anthocyanins have been tested. We have designed an intervention diet (Fig. 4), which we believe delivers the most balanced profile of the major fruit anthocyanins ( $15 \mathrm{~g}$ of blueberry, $5 \mathrm{~g}$ of blackberry, $30 \mathrm{~g}$ cranberry, $20 \mathrm{~g}$ of grape, $5 \mathrm{~g}$ of raspberry and $25 \mathrm{~g}$ of strawberry; $100 \mathrm{~g}$ mixed fruit portion). This diet contains a balanced amount of the 6 anthocyanidins delphinidin $(9.39 \mathrm{mg} / 100 \mathrm{~g} \mathrm{FW})$, cyanidin $(14.44 \mathrm{mg} / 100 \mathrm{~g} \mathrm{FW})$, petunidin $(5.09 \mathrm{mg} / 100 \mathrm{~g} \mathrm{FW})$, pelargonidin $(8.97 \mathrm{mg} / 100 \mathrm{~g}$ $\mathrm{FW})$, peonidin $(2.96 \mathrm{mg} / 100 \mathrm{~g} \mathrm{FW})$ and malvidin $(12.21 \mathrm{mg} / 100 \mathrm{~g} \mathrm{FW})$, and may be utilised to assess the full impact of anthocyanins on the vascular system, the brain and against cancer cell proliferation in human studies. This diet represents an excellent basis for a future mixed anthocyanidin intervention diet. In addition, analyses should be performed before intervention to check that the designed diet reflects the balanced anthocyanidin profile. Furthermore, the use of food and test materials that are analytically well characterized and standardized with regard to anthocyanin content will enable significant advancement and allow high-quality data comparison, and the potential impact of dietary anthocyanins on health could be more comprehensively evaluated [29].

\section{References}

[1] G. Borges, A. Degeneve, W. Mullen and A. Crozier, Identification of flavonoid and phenolic antioxidants in black currants, blueberries, raspberries, red currants, and cranberries, J Agric Food Chem 58 (2010), 3901-3909.

[2] N.A. Nyman and J.T. Kumpulainen, Determination of anthocyanidins in berries and red wine by high-performance liquid chromatography, J Agric Food Chem 49 (2001), 4183-4187.

[3] D.C. Grant, R.J. Helleur, D.C. Grant and R.J. Helleur, Rapid screening of anthocyanins in berry samples by surfactant-mediated matrixassisted laser desorption/ionization time-of-flight mass spectrometry, Rapid Commun Mass Spectrom 22 (2008), $156-164$.

[4] K.R. Maatta-Riihinen, A. Kamal-Eldin, A.R. Torronen, K.R. Maatta-Riihinen, A. Kamal-Eldin and A.R. Torronen, Identification and quantification of phenolic compounds in berries of Fragaria and Rubus species (family Rosaceae), J Agric Food Chem 52 (2004), $6178-6187$. 
[5] K. Ogawa, H. Sakakibara, R. Iwata, T. Ishii, T. Sato, T. Goda, K. Shimoi, S. Kumazawa, K. Ogawa, H. Sakakibara, R. Iwata, T. Ishii, T. Sato, T. Goda, K. Shimoi and S. Kumazawa, Anthocyanin composition and antioxidant activity of the Crowberry (Empetrum nigrum) and other berries, J Agric Food Chem 56 (2008), 4457-4462.

[6] R. Slimestad, H. Solheim, R. Slimestad and H. Solheim, Anthocyanins from black currants (Ribes nigrum L.), J Agric Food Chem 50 (2002), 3228-3231.

[7] X. Wu, L. Gu, R.L. Prior, S. McKay, X. Wu, L. Gu, R.L. Prior and S. McKay, Characterization of anthocyanins and proanthocyanidins in some cultivars of Ribes, Aronia, and Sambucus and their antioxidant capacity, J Agric Food Chem 52 (2004), 7846-7856.

[8] J. Ginè Bordonaba, G.A. Chope and L.A. Terry, Maximising blackcurrant anthocyanins: temporal changes during ripenin and storage in different genotypes, J Berry Res 1 (2010), 73-80.

[9] F. Mattivi, R. Guzzon, U. Vrhovsek, M. Stefanini, R. Velasco, F. Mattivi, R. Guzzon, U. Vrhovsek, M. Stefanini and R. Velasco, Metabolite profiling of grape: Flavonols and anthocyanins, J Agric Food Chem 54 (2006), 7692-7702.

[10] S. Pati, M.T. Liberatore, G. Gambacorta, D. Antonacci, E. La Notte, S. Pati, M.T. Liberatore, G. Gambacorta, D. Antonacci and E. La Notte, Rapid screening for anthocyanins and anthocyanin dimers in crude grape extracts by high performance liquid chromatography coupled with diode array detection and tandem mass spectrometry, J Chromatogr 1216 (2009), 3864-3868.

[11] M. Battino, J. Beekwilder, B. Denoyes-Rothan, M. Laimer, G.J. McDougall and B. Mezzetti, Bioactive compounds in berries relevant to human health, Nutr Rev 67 (2009), S145-S150.

[12] L.S. Wang and G.D. Stoner, Anthocyanins and their role in cancer prevention, Cancer Lett 269 (2008), 281-290.

[13] C. Mohn, M. Baum and E. Richling, Antioxidative efficiency of an anthocyanin rich bilberry extract in the human colon tumor cell lines Caco-2 and HT-29, J Berry Res 1 (2010), 25-33.

[14] S. de Pascual-Teresa, D.A. Moreno and C. Garcia-Viguera, Flavanols and anthocyanins in cardiovascular health: a review of current evidence, Int J Mol Sci 11 (2010), 1679-1703.

[15] A. Basu, M. Rhone and T.J. Lyons, Berries: emerging impact on cardiovascular health, Nutr Rev 68 (2010), 168-177.

[16] J.P. Spencer, Nutrients and brain health: an overview, Genes Nutr (2009).

[17] J.P. Spencer, Flavonoids and brain health: multiple effects underpinned by common mechanisms, Genes Nutr 4 (2009), $243-250$.

[18] A.L. Macready, O.B. Kennedy, J.A. Ellis, C.M. Williams, J.P. Spencer and L.T. Butler, Flavonoids and cognitive function: a review of human randomized controlled trial studies and recommendations for future studies, Genes Nutr 4 (2009), 227-242.

[19] P.J. Mink, C.G. Scrafford, L.M. Barraj, L. Harnack, C.P. Hong, J.A. Nettleton and D.R. Jacobs, JJr., Flavonoid intake and cardiovascular disease mortality: a prospective study in postmenopausal women, Am J Clin Nutr 85 (2007), 895-909.

[20] T.H. Rissanen, S. Voutilainen, J.K. Virtanen, B. Venho, M. Vanharanta, J. Mursu and J.T. Salonen, Low intake of fruits, berries and vegetables is associated with excess mortality in men: the Kuopio Ischaemic Heart Disease Risk Factor (KIHD) Study, J Nutr 133 (2003), 199-204.

[21] H.D. Sesso, J.M. Gaziano, D.J. Jenkins and J.E. Buring, Strawberry intake, lipids, C-reactive protein, and the risk of cardiovascular disease in women, J Am Coll Nutr 26 (2007), 303-310.

[22] O.K. Chun, S.J. Chung, K.J. Claycombe and W.O. Song, Serum C-reactive protein concentrations are inversely associated with dietary flavonoid intake in U.S. adults, J Nutr 138 (2008), 753-760.

[23] M.P. Kahkonen and M. Heinonen, Antioxidant activity of anthocyanins and their aglycons, J Agric Food Chem 51 (2003), $628-633$.

[24] X. Wu and R.L. Prior, Systematic identification and characterization of anthocyanins by HPLC-ESI-MS/MS in common foods in the United States: fruits and berries, J Agric Food Chem 53 (2005), 2589-2599.

[25] J. Kuhnau, The flavonoids. A class of semi-essential food components: their role in human nutrition, World Rev Nutr Diet 24 (1976), $117-191$.

[26] C.A. Williams and R.J. Grayer, Anthocyanins and other flavonoids, Nat Prod Rep 21 (2004), 539-573.

[27] A. Crozier, I.B. Jaganath and M.N. Clifford, Dietary phenolics: chemistry, bioavailability and effects on health, Nat Prod Rep 26 (2009), 1001-1043.

[28] X. Wu, G.R. Beecher, J.M. Holden, D.B. Haytowitz, S.E. Gebhardt and R.L. Prior, Concentrations of anthocyanins in common foods in the United States and estimation of normal consumption, J Agric Food Chem 54 (2006), 4069-4075.

[29] H. Schroeter, C. Heiss, J.P. Spencer, C.L. Keen, J.R. Lupton and H.H. Schmitz, Recommending flavanols and procyanidins for cardiovascular health: current knowledge and future needs, Mol Aspects Med 31 546-557.

[30] M.M. Rahman, T. Ichiyanagi, T. Komiyama, Y. Hatano and T. Konishi, Superoxide radical- and peroxynitrite-scavenging activity of anthocyanins; structure-activity relationship and their synergism, Free Radic Res 40 (2006), 993-1002.

[31] C.D. Kay, P.A. Kroon and A. Cassidy, The bioactivity of dietary anthocyanins is likely to be mediated by their degradation products, $M o l$ Nutr Food Res 53Suppl 1 (2009), S92-S101.

[32] A. Karlsen, L. Retterstol, P. Laake, I. Paur, S. Kjolsrud-Bohn, L. Sandvik and R. Blomhoff, Anthocyanins inhibit nuclear factorkappaB activation in monocytes and reduce plasma concentrations of pro-inflammatory mediators in healthy adults, J Nutr 137 (2007), 1951-1954. 